\title{
Trade-off between stochastic and deterministic processes shifts from soil to leaf microbiome of tea plant
}

\section{Ping Xu}

Zhejiang University

\section{Erinne Stirling}

Zhejiang University

\section{Wenbing Li}

Hangzhou Normal University

Xiaofei Lv

China Jiliang University

\section{Haiyan Chen}

Zhejiang University

\section{Anan $\mathrm{Xu}$}

Zhejiang University

Wanyi Lai

Zhejiang University

Yuefei Wang

Zhejiang University

Xingmei Liu

Zhejiang University

Bin Ma ( $\sim$ bma@zju.edu.cn )

Zhejiang University https://orcid.org/0000-0003-4807-4992

Jianming Xu

Zhejiang University

\section{Research}

Keywords: Camellia sinensis, distance dispersal, endophytic bacteria, environmental limitation, cooccurrence pattern, stochastic process

Posted Date: May 18th, 2020

DOI: https://doi.org/10.21203/rs.3.rs-28737/v1 
License: (c) (i) This work is licensed under a Creative Commons Attribution 4.0 International License. Read Full License 


\section{RESEARCH}

\section{Trade-off between stochastic and deterministic processes shifts from soil to leaf microbiome of tea plant}

Ping $\mathrm{Xu}^{1}$, Erinne Stirling ${ }^{2}$, Wenbing $\mathrm{Li}^{3}$, Xiaofei $\mathrm{Lv}^{4}$, Haiyan $\mathrm{Chen}^{1}$, Anan $\mathrm{Xu}^{1}$, Wanyi Lai ${ }^{1}$, Yuefei Wang ${ }^{1}$, Xingmei $\mathrm{Liu}^{2}$, Bin $\mathrm{Ma}^{2 *}$ and Jianming $\mathrm{Xu}^{2}$

${ }^{*}$ Correspondence: bma@zju.edu.cn

${ }^{2}$ College of Environmental and Natural Resource Sciences, Zhejiang Provincial Key Laboratory of Agricultural Resources and Environment, Zhejiang University, 310058 Hangzhou, China

Full list of author information is available at the end of the article

\begin{abstract}
Background: Plant microbiome is thought to play an important role in promoting plant health and production. However, even though the microbiomes in various compartments have been widely investigated, the association between above and belowground compartments of plants remain unclear. Tea is a globally popular beverage due to its flavor and health benefits associating with secondary metabolites; the microbiomes of tea plant (Camellia sinensis) play a significant role in the production of these secondary metabolites. Here, we investigated the microbiomes of bulk and rhizosphere soils, roots and leaves of $C$. sinensis collected from tea plantations across over $2000 \mathrm{~km}$ to investigate the association and driving mechanisms for microbiomes in the compartments.

Results: Camellia sinensis microbiomes differed between the compartments with $\alpha$-diversity gradually decreasing from soils to roots and leaves. The core leaf microbiome comprised Bacilli, Sphingobacteriia and $\alpha$-Proteobacteria, which we suggest might ascendingly migrate from soils to leaves. Microbial community assembly processes were dominated by deterministic processes in bulk and rhizosphere soils; these assembly processes were dominated by stochastic processes in roots and leaves. Dispersal limitation was stronger in old leaves than in other compartments. Amino acids were also critical drivers for environmental selection. The microbiomes in $C$. sinensis roots and leaves possessed a lower intensity of microbial associations and more negative microbial associations than in bulk and rhizosphere soils, suggesting that the contribution of microbial interactions varied in different compartments.
\end{abstract}

Conclusion: In summary, there is a trade-off between stochastic and deterministic processes in microbiomes community assembly along from soil to leaf of $C$. sinensis. These results provide valuable information for understanding the associations and driving mechanism of microbiomes in various $C$. sinensi compartments, which could be used to predict $C$. sinensis microbiome and harness its power to improve tea production and quality.

Keywords: Camellia sinensis; distance dispersal; endophytic bacteria; environmental limitation; co-occurrence pattern; stochastic process

\section{Background}

2 Tea is one of the most popular non-artificial and non-alcoholic beverages consumed across the world due to its unique flavor and potential health benefits[1]. Hence, the tea plant Camellia sinensis is a critical horticultural crop with a global total planting area of 4.89 million hectares[2]. Tea flavors are mainly attributed to 
secondary metabolite characteristics such as flavonoids, theanine and caffeine in $C$. sinensis leaves[3]. These secondary metabolites are also in high demand in the pharmaceuticals and naturopathy industries due to their health benefits for human[4]. Flavonoids are a group of plant polyphenol secondary metabolites that are synthesized within the general phenylpropanoid pathway[5]. Numerous pieces of evidence have revealed that flavonoids have beneficial effects against diseases such as cancer, neurodegenerative diseases, autoimmune diseases and cardiovascular diseases[6]. Theanine accumulates in tea plants but is rarely produced in other plants and is an important indicator in the qualitative assessment of green tea[3]. This compound is a nonproteinogenic amino acid with proven health benefits such as neuroprotection, relaxation and enhancement of cognitive performance[7]. Caffeine is a purine alkaloid that is abundant in tea leaves[3]; it is a well-known central nervous system stimulant that can also lower blood pressure and be used for diabetes prevention[7]. The microbiomes of $C$. sinensis play a significant role in the production of these aforementioned secondary metabolites through two primary mechanisms[2]. Firstly, endophytic and rhizosphere bacteria promote the production of secondary metabolites in C. sinensis through plant growth hormone production, phosphate solubilization, nutrient acquisition and N2 fixation[4]. Secondly, the characteristic secondary metabolites, or their precursors, can be produced by endophytic bacteria associated with $C$. sinensis . For example, the endophytic bacteria Luteibacter, isolated from C. sinensis, has shown strong biocatalytic activity for converting both glutamine and ethylamine to theanine[8]. Many bacterially originating enzymes can potentially catalyze flavonoid modification, leading to the construction of natural and novel flavonoid derivatives9. Endophytic bacteria also have complex interactions with the metabolism of secondary metabolites in C. sinensis as flavonoids possess antibacterial activity[10] and caffeine can synergistically enhance the antibacterial activity of other compounds such as $\alpha$-dicarbonyl, glyoxal, methylglyoxal, and diacetyl[11]. The antibacterial activity of flavonoids and caffeine could therefore regulate endophytic bacterial communities[12]. In contrast, endophytic bacteria can also degrade flavonoids via deglycosylation[13] and degrade caffeine via demethylation and oxidation pathways[14]. The microbiomes of $C$. sinensis are also a key factor in the fermentation processes of various tea products[15]. Accordingly, understanding the assembly processes for $C$. sinensis microbiomes is essential for improving the production and quality of tea products.

Endophytic microbiomes are transmitted through seed dispersal or recruited from a plant's surrounding environment[3]. Root microbiomes enter the host plant through the cracks formed in the lateral root junctions or through wounds caused by microbe or nematode phytopathogens and quickly spread to the endorhizosphere[16]. Entry of endophytic bacteria in plant roots also occurs via root hairs and the spaces between epidermal cells[16]. Utilizing a dynamic infection process, the endophytic bacteria Rhizobia have been found to ascendingly migrate from roots to leaves in rice plants, where they transiently grow to large local populations[17]. Plant associated microbiomes in roots and rhizospheres also benefit host plants by enhancing nutritional acquisition and improving resistance to pathogenic infections[18]. Understanding the community assembly of plant microbiomes is crucial for managing both ecological function and crop production[19]. 
Progress in plant microbiomes has been made toward specific compartments, such as rhizosphere, roots, leaves and phyllosphere in certain model and crop plant species, such as Arbidopsis[20, 21], rice[22], wheat[23], barley[24], maize[25, 26], soybean[27] and citrus[28]. However, the associations of plant microbiomes across different compartments from soils to leaves is needed to understand microbial functions for host plants in supporting sustainable agriculture and healthy earth ecosystems.

In this study, we investigated $C$. sinensis microbiomes by unravelling the underlying associations along soil to leaf microbiomes. China is the dominant country tea production with a total planting area of approximately 3 million hectares, which accounts for $63 \%$ of the global area for tea plantation[2]. We collected microbiomes of $C$. sinensis, including bulk and rhizosphere soils, roots, young leaves and old leaves, from the dominant tea planting regions in China. We then determined the contribution of stochastic and deterministic processes in various plant and soil compartments and assessed their associations.

\section{Methods}

\section{Sample collection}

To capture biogeographical differences in $C$. sinensis microbiomes, we collected samples from 45 locations spanning all 15 tea planting provinces in China (Fig. 1a). The samples were collected from diverse $C$. sinensis varieties, soil types and climate types (Table S1); soil characteristics are listed in Data file S1. Five compartments (bulk soil, rhizosphere, roots, young leaves and old leaves) were collected at each plantation using the following protocol. At least 15 healthy $C$. sinensis plants (10 $\mathrm{m}$ of separation) were selected from each tea plantation for sample collection. For each plant, $200 \mathrm{~g}$ young leaves and $200 \mathrm{~g}$ old leaves were clipped, the top $5 \mathrm{~cm}$ of soil was removed and fine roots (approximately1 mm diameter) from a depth of $5-20 \mathrm{~cm}$ were collected. The roots were removed from the soil with a shovel and then gently shaken to remove the soil not tightly attached to the roots. Soil tightly attached to the roots was termed rhizosphere soils. Soil from the same 5-20 cm depth without any roots near the selected trees was termed bulk soil. All subsamples for each compartment were mixed throughout to create a representative sample for each plantation. Samples were transported to the laboratory within 3 days and stored at $-20{ }^{\circ}$ Cuntil DNA extraction.

\section{DNA extraction and sequencing}

Rhizosphere and bulk soil DNA was extracted from each sample using an MP FastDNA soil extraction kit (MP Laboratories Inc. Carlsbad, CA, USA). Root and leaf DNA was extracted from each sample using a MinkaGene Plant DNA Kit (mChip BioTech, Guangzhou, China). Extracting protocols followed the manufacturer's instructions with some modifications. DNA quality and quantity were determined by using a NanoDrop device (Thermo Scientific, Wilmington, DE). The $16 \mathrm{~S}$ rDNA amplicon library preparation and sequencing were performed according to the manufacturer's protocol at Novogene, China. For the amplicon library preparation, amplification of $16 \mathrm{~S}$ rDNA V5-V7 region fragments was performed using primers $799 \mathrm{~F}$ and $1193 \mathrm{R}[29]$. After quality control and quantification and normalization of the DNA libraries, 250-bp paired-end reads were generated using an Illumina NovaSeq 6000 system (Illumina inc, San Diego, US) according to the manufacturer's instructions. 
Amplicon data analysis

Microbial community composition was determined by sequencing $16 \mathrm{~S}$ rDNA amplicons. The high-quality paired-end reads of the $16 \mathrm{~S} \mathrm{V5-V7}$ region were merged using USEARCH v11[30]. OTUs were obtained using the UPARSE pipeline based on the merged sequences[30]. OTU taxonomy was generated using representative sequences of each OTU and aligned against the RDP II databases. OTUs and merged sequences that were defined as originating from unknown, chloroplast, mitochondria or plant sources were removed.

\section{Diversity and ordination}

$\alpha$-diversity was calculated for each sample using the Shannon-Wiener index based on the normalized OTU abundance table using the binomial method[31]. Community dissimilarity analysis between samples was performed using Principal Coordinate Analysis (PCoA) with unweighted UniFrac distances.

\section{Core microbiota identification}

The core microbiota for C. sinensis was defined as cohort of OTUs present in all 225 samples. Unique core microbiota for each compartment was defined as the OTUs present in all 45 samples of the corresponding compartment and also absent in all other compartments.

\section{Source tracker analysis}

The potential source of microbial compositions among various compartments was estimated using SourceTracker[32].

\section{Enrichment and depletion from soils to leaves}

OTU enrichment and depletion were calculated using the DESeq function of the $\mathrm{R}$ package DESeq2 based on the negative binomial distribution algorithm[33].

\section{Normalized stochastic ratio estimation}

Normalized stochasticity ratio (NST) of communities in samples and null model was calculated with tNST function of the R package NST[34]. The null model calculation was repeated 10 times.

\section{Nestedness estimation}

Community nestedness at various taxonomic ranks was calculated with nestednodf function in vegan package. The corresponding nestedness using a null model was calculated based on a matrix generated with the permatfull function in vegan package.

\section{Dispersal limitation}

Community similarity geographic decay was tested by estimating the relationship between community similarity and geographic distance. Dispersal limitation was tested using correlations between community similarity and geographic distance under a partial correlation condition for environmental properties using partial mantel statistic with mantel.partial function in vegan package. The spatial correlation of communities along geographic distance was determined with Mantel correlogram using mantel.correlog function in vegan package. 


\section{Environmental selection}

Amino acid and catechin concentrations in roots, young leaves and old leaves, the physiochemical properties of rhizosphere and bulk soils, and the concentrations of 8 heavy metals were measured in all samples (Data file S1-S5). The impact of these environmental factors on microbial communities for corresponding compartments was estimated using constrained corresponding analysis with cca function in vegan.

\section{Microbial co-occurrence network}

Microbial co-occurrence networks for each compartment were constructed based on a spearman correlation matrix for the OTUs presenting in each compartment. In order to avoid the taxon number biases, each community dataset was trimmed to the dominant 400 taxa. p-values were adjusted for multiple tests using the Benjamini and Hochberg FDR controlling procedure with the R package multtest[35]. Direct correlation dependencies were distinguished using the network enhancement method[36] and thresholds values for Spearman cutoffs were determined by Random matrix theory (RMT) method[37]. Network properties were calculated with the igraph package for $\mathrm{R}[38]$.

\section{Statistics}

All the statistics were performed using R 3.6.0[39]. Significant difference of $\alpha$ diversity across compartments were determined using one-way analysis of variance (ANOVA) and Tukey's 'Honest Significant Difference'(HSD) method. The significant differences of community dissimilarity were determined using analysis of similarities (ANOSIM). Significant differences of NST between communities in samples and null model were determined using one-tailed one-sample t-tests.

\section{Results}

Core microbiota of $C$. sinensis microbiomes from soil to leaves

A total of 225 samples from 45 tea plantations in prominent tea-producing regions of China (Fig. 1a, Table S1) were collected to explore the microbiomes of bulk soil, rhizosphere soil (hereafter 'rhizosphere'), roots, young leaves and old leaves. These 45 locations included 4 soil types and two climate types, with contrasting altitude (20-1600 m), pH (4.1-7.4). We sequenced the 16S rDNA amplicon for each compartment of all samples (approximately 79.8 million high-quality sequence tags). An average of 0.27 million tags were generated for each sample after removal of sequences associated with chloroplast and mitochondrial DNA.

Microbial $\alpha$-diversity (Shannon-Wiener) decreased with compartment distance from soil ( $A N O V A, D F=4, F=75.3, P<0.001$ ) (Fig. 1b). $\beta$-diversity (UniFrac distance) also revealed that the community composition differentiated between soils (bulk and rhizosphere), roots and leaves (young and old) (ANOSIM, $R=0.76, P=$ 0.001) (Fig. 1c). Core taxa were defined as the genera present in all compartment specific samples and none of the other samples; the number of core operational taxonomic units (OTUs) decreased sequentially from bulk soils to rhizosphere, root, old leaves, and young leaves (Fig. 1d). Bulk soil, rhizosphere, roots, young leaves and old leaves contained 140, 138, 96, 28 and 13 core OTUs, respectively (Fig. 1e). There were a further 10 generalist OTUs present in all compartments. Proteobacteria, Actinobacteria and Acidobacteria dominated multiple compartments. 


\section{Enrichment and depletion of $C$. sinensis microbiomes from soils to leaves}

The SourceTracker analysis revealed substantial similarities within the two leaf compartments and within the two soil compartments, and also indicates that there are rare exchanges between roots and leaves, indicating partitioning between above and belowground microbiomes (Fig. 2a). This transfer bottleneck can be observed as a $34 \%$ similarity between the rhizosphere and roots but only a $5 \%$ similarity between roots and old leaves. When genera are compared sequentially across the compartments, each ascension in a compartment (e.g. from soil to rhizosphere or old leaf to young leaf) lead to substantially more depleted OTUs than enriched OTUs (Fig $2 \mathrm{~b}$ ). Depletion ratios (depletion/enrichment) ranged from 0.5 in rhizosphere/bulk soil to 7.5 for both old leaf/root and root/rhizosphere. Sphingomonas, Methylobacterium and Burkholderia were frequently associated with these changes (Fig. 2c). These results show strong filtering effects from rhizosphere samples to roots and from roots to leaves.

\section{Community assembly mechanisms of $C$. sinensis microbiomes from soils to leaves}

Community assembly normalized stochasticity ratio (NST) increased from $40 \%$ in bulk soil to more than $70 \%$ in leaves (Fig. 3a). Nestedness was significant for rhizosphere (t-tests, $P<0.05)$ and bulk soil microbiomes (t-tests, $P<0.05)$ but non-significant for root and leaf microbiomes (t-tests, $P>0.05$ ) at all ranks from genus to phylum (Fig. 3b). Microbiomes in soils, roots and leaves were nested as subsets of rhizosphere microbiomes and show distinct patterns in species richness (Fig 3c). When considered using Bray-Cutis similarity and after controlling the impact of the environmental matrix using Partial Mantel tests, all compartments were negatively correlated with geographic distance (Fig. 3d). This response was particularly strong in old leaves; Mantel correlograms show that only this compartment linearly changed with geographic distance (Fig. 3e). The environmental selection effect of physiochemical properties in rhizosphere and bulk soils, amino acids and catechin in roots and leaves, and heavy metals on microbial communities was assessed using constrained corresponding analysis (Fig. 3f). Explanation proportions of the constrained axes suggest that the contribution of environmental selection decreased from leaves and roots to rhizosphere and bulk soils. The microbial community structures in plant tissues were closely associated with amino acids in young leaves, old leaves and roots; microbial community structures in old leaves were additionally closely associated with metals. Bulk soil microbial community structures were closely associated with environmental variables such as altitude, soil $\mathrm{pH}$, phosphorus, carbon and metals while rhizosphere microbial communities were closely associated with soil $\mathrm{pH}$ and nitrogen variables.

\section{Microbial co-occurrence networks of $C$. sinensis microbiomes from soils to leaves}

Potential interaction patterns within microbial communities were compared by inferring co-occurrence networks using the abundance matrix of the 1000 most abundant OTUs. The number of links, representing the intensity of potential microbial interactions, was the largest in bulk and rhizosphere soils, followed by roots and young leaves, and was the smallest in old leaves (Fig. 4). The network diameter, representing the longest path in a network, was the longest in young and shortest in 
old leaves; transitivity, representing network modularity, was highest in roots and lowest in young leaves (Table S2). Although plant tissues had fewer links, negative links in tissue microbiomes were more abundant than in rhizosphere and bulk soils (Fig. 4). The co-occurrence networks also displayed different taxon assortativity (Table S2). Overrepresented links include Proteobacteria to Firmicutes in young leaves, Proteobacteria to Bacteroidetes in old leaves, Proteobacteria to Actinobacteria and Proteobacteria to Acidobacteria in roots, rhizosphere and bulk soils (Fig. 4). Intra-Acidobacteria links were substantially overrepresented in rhizosphere and bulk soils.

\section{Discussion}

Microbiomes are important mediators in maintaining the overall health and production capacity of their plant hosts; however, little is known about the associations across microbiomes in various plant compartments. Here we have collected C. sinensis microbiomes from samples across China to assess community assembly mechanisms. The key finding of this research is that there is a trade-off between stochastic and deterministic processes in above and belowground C. sinensis microbiomes (Fig. 5). This finding allows us to use community assembly mechanisms to predict $C$. sinensis microbiomes, which offers fundamental information to explore the role of microbiomes in improving tea productivity and quality.

The NST values suggest that microbial community assembly processes were dominated by deterministic processes in bulk soil and rhizosphere samples, and by stochastic processes in root and leaf samples; this result is supported by the microbiome nestedness patterns and may due to a decrease in environmental selection strength from leaf to root to soil. Belowground microbes face environmental selection pressures of oxygen content, nutrient supply and space limitation while phyllosphere microbes are more randomly distributed on leaves through atmospheric deposition, seed-associated dispersion and animal sources[40]. The increasing community similarity from leaf to root and soil in our study supports this inference as it indicates an increasing environmental selection strength[34]. It is generally accepted that stochasticity dominates the early stages of community establishment while deterministic processes become progressively important over time[41]. Thus, the decreased contribution of stochastic processes from leaf to root and soil may also result from time since community establishment.

Deterministic processes mainly consist of dispersal limitation, environmental selection and biological interactions[42]. The importance of dispersal limitation and environmental selection as ecological processes shaping tea microbiomes can be observed in the significant decline of microbial similarity with geographic distance. The associations between microbial communities and geographic distance after controlling for the impact of the environmental matrix suggest a significant impact of dispersal limitation on all compartment microbiomes. This impact may be due to the interactions of drift and dispersal limitation among leaves, rhizosphere and soil. As the movement of a single taxon from air or animal to leaves is a random event, leaf microbial composition may largely depend on stochastic migration processes. However, dispersal limitation is also important in maintaining phyllosphere community structure as microbes are host-associated; therefore the successful establishment of a newly arrived taxon is more limited than new taxa in soils[43]. In 
addition, drift processes are more important when $\alpha$-diversity and microbial community abundances are low[44]. Accordingly, the low Shannon-Wiener index for leaf microbiomes suggests that both drift and dispersal limitation are important ecological processes for shaping the microbial assembly of leaf microbiomes; thus, both drift and dispersal limitation strengthen the geographic community similarity decay in old leaves. However, this drift effect may be weaker in new leaves and is masked by the strong selection pressures in soil[45].

The discrepancy in driving factors for environmental selection from soils to leaves suggests that divergent environmental selection processes are active in the compartments. It is not surprising to find that soil $\mathrm{pH}$, the best predictor of soil bacterial and archaeal community composition across global[46], regional[47] and local scales[48], is an important driver for microbiomes in both the rhizosphere and bulk soil. In addition to soil $\mathrm{pH}$, soil organic carbon quality and quantity and nitrogen and phosphorus availability also have notable influences on soil microbial community structure[49]. The results here indicate that microbial communities were closely associated with dissolved organic carbon and available phosphorus in bulk soils and dissolved organic nitrogen (DON) in rhizosphere. Organic carbon substrates and phosphorus compounds exhibit enormous ranges and are persistent abiotic stressors affecting microbial survival and growth in soils[49]. However, carbon and phosphorus limitation in the rhizosphere can be alleviated by root exudates and soil $\mathrm{pH}$ neutralization[50]. Decreased nitrogen availability in the rhizosphere also induces microbial DON stress16. The significant impact of $\mathrm{Cd}$ on microbiomes in bulk soil relative to rhizosphere samples may be due to the alleviation of metal toxicity by increased rhizosphere soil $\mathrm{pH}[51]$.

The associations between amino acids and endophytic bacteria in roots and leaves suggest that plant tissue amino acids play a vital role in endophytic bacterial community assembly. Endophytic bacteria produce alkaloids as secondary metabolites; these compounds have diverse potentials as anti-fungal and -viral agents[3]. Most alkaloids are derived from amines produced by the decarboxylation of amino acids[3], such as tyrosine in roots and young leaves, and lysine in old leaves. There was an association between endophytic bacteria and metals such as $\mathrm{Cd}, \mathrm{Pb}$ and $\mathrm{Cu}$ in old leaves, and metals such as $\mathrm{Ni}$ in young leaves. Endophytic bacteria can potentially bioaccumulate metals from contaminated mediums as shown by LK11 reprograming its amino acids and proteomic expressions to maintain steady growth during Cd stress[52]. Phytohormone producing endophytic bacterium could therefore be an ideal approach to increase the phytoextraction potential of metal contamination bioremediation plants. The association between $\mathrm{Ni}$ and young leaves is unsurprising as it is a urease activator, which is required for young tissue growth[53].

The topological properties of microbial co-occurrence networks indicate different microbial interaction contributions for microbial community assembly in the compartments. The decreasing gradient in linking intensity from soils to leaves suggests that potential microbial interactions in soils are more complex than those in plant tissues. Although the linking intensity was higher for young leaves than old leaves, the longer network diameter for young leaves suggests that the interaction is still inefficient and that young leaf microbial communities may not be capable of reaching a stable status. Co-occurrence network modules indicate potential niche 
numbers in corresponding microbial communities[54]. The low modularity for young leaves indicates sparse niches in this compartment while the high modularity for root indicates the opposite. The high number of negative associations in plant tissues suggests a high prevalence of adverse interactions as negative associations in microbial communities represents potential antagonistic relationships[55].

Core microbiota are the consequence of long-term environmental selection in specific environments. The core microbiota in leaves of $C$. sinensis, including Bacilli, Sphingobacteriia and $\alpha$-Proteobacteria, have been widely identified as endophytic bacteria in pine leaves[56], maple leaves[57] and several plant species found in Tallgrass Prairie communities[58], suggesting that these microbial taxa are adapted to leaf tissue environments. Genomic analyses of plant-associated $\alpha$-Proteobacteria has shown that they possess a remarkable number of regulators, sugar transporters, metabolic enzymes and nodulation genes[59]. Although the pattern of depletion was not consistent between ascending compartments, Methylobacterium ( $\alpha$ Proteobacteria) and Sphingomonas (Sphingobacteriia) were consistently enriched in each compartment from soils to leaves. This enrichment effect also explains the formation of core microbiota in $C$. sinensis leaves; overrepresented taxon-associations in each microbial co-occurrence network were mainly associated with the core microbiota in corresponding compartments, indicating an essential role of core microbiota in community assembly.

\section{Conclusion}

In summary, the present study provides an overview of $C$. sinensis microbiome in compartments from soils to leaves. With this study, we have shown that C. sinensis microbiomes gradually changed along the compartments from soils to leaves in term of $\alpha$ - and $\beta$-diversity. Moreover, we find that microbial community assembly processes were dominated by deterministic processes in bulk and rhizosphere soils; these assembly processes were dominated by stochastic processes in roots and leaves. The driving mechanisms for community assembly allows us to use community assembly mechanisms to predict $C$. sinensis microbiomes. In addition, the association between amino acids and endophytic leaf microbiomes provide insight into the roles of tea microbiomes in improving the productivity and quality of tea production. While this study provides a comprehensive analysis of associations across $C$. sinensis microbiome in compartments from soils to leaves, our understanding is still in its infancy. Although these analyses provide insight into the driving mechanisms for community assembly, the roles of tea microbiomes in improving the productivity and quality of tea production can only be fully understood by deciphering the underlying relationships between endophytic leaf microbiomes and $C$. sinensis's secondary metabolites.

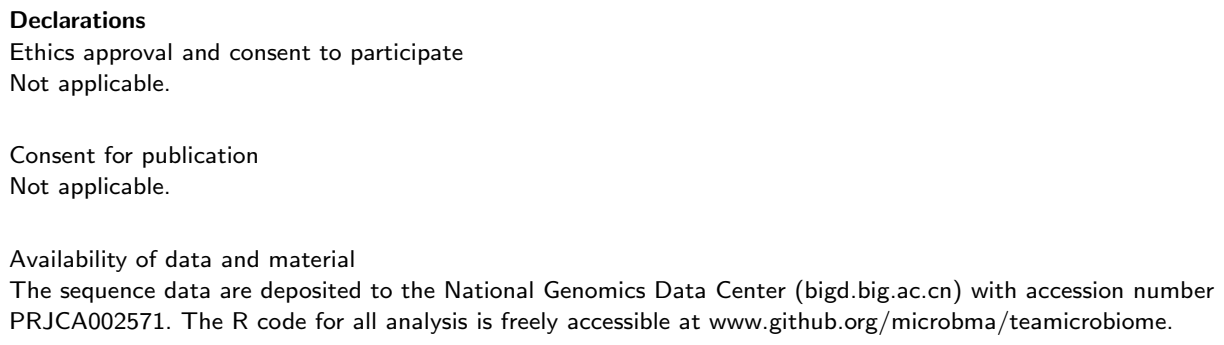


Competing interests

The authors declare that they have no competing interests.

Funding

This research was financially supported by the National Natural Science Foundation of China (41991334) and the Zhejiang Provincial Natural Science Foundation of China (LD19D060001).

Authors' contributions

PX, WL, YW, BM, and JX designed the study. PX, ES, and BM wrote the manuscript. PX, XL, HC, WL, AX, WL, and $\mathrm{XL}$ collected the samples and performed physicochemical analysis. $\mathrm{ES}, \mathrm{WL}, \mathrm{XL}$, and BM performed data analysis. All authors read and approved the manuscript.

Acknowledgements

Not applicable.

Author details

${ }^{1}$ Department of Tea Science, Zhejiang University, 310058 Hangzhou, China. ${ }^{2}$ College of Environmental and Natural Resource Sciences, Zhejiang Provincial Key Laboratory of Agricultural Resources and Environment, Zhejiang University, 310058 Hangzhou, China. ${ }^{3}$ Key Laboratory of Hangzhou City for Ecosystem Protection and Restoration, College of Life and Environmental Sciences, Hangzhou Normal University, 311121 Hangzhou, China.

${ }^{4}$ Department of Environmental Engineering, China Jiliang University, 310018 Hangzhou, China.

\section{References}

1. Wei C, Yang H, Wang S, Zhao J, Liu C, Gao L, et al. Draft genome sequence of Camellia sinensis var. sinensis provides insights into the evolution of the tea genome and tea quality. Proc Natl Acad Sci U S A. 2018;115:E4151-8.

2. Yan X, Wang Z, Mei Y, Wang L, Wang X, Xu Q, et al. Isolation, diversity, and growth-promoting activities of endophytic bacteria from tea cultivars of Zijuan and Yunkang-10. Front Microbiol 2018;9:1848.

3. Li C-F, Zhu Y, Yu Y, Zhao Q-Y, Wang S-J, Wang X-C, et al. Global transcriptome and gene regulation network for secondary metabolite biosynthesis of tea plant (Camellia sinensis). BMC Genomics. 2015;16:560.

4. Singh M, Kumar A, Singh R, Pandey KD. Endophytic bacteria: a new source of bioactive compounds. 3 Biotech. 2017;7:315.

5. Singh HP, Ravindranath SD, Singh C. Analysis of tea shoot catechins: spectrophotometric quantitation and selective visualization on two-dimensional paper chromatograms using diazotized sulfanilamide. J Agric Food Chem. 1999;47:1041-5.

6. Higdon JV, Frei B. Tea catechins and polyphenols: health effects, metabolism, and antioxidant functions. Crit Rev Food Sci Nutr. 2003;43:89-143.

7. Alagawany M, Abd El-Hack ME, Saeed M, Naveed M, Arain MA, Arif M, et al. Nutritional applications and beneficial health applications of green tea and I-theanine in some animal species: A review. J Anim Physiol Anim Nutr. 2020;104:245-56.

8. Sun J, Chang M, Li H, Zhang Z, Chen Q, Chen Y, et al. Endophytic bacteria as contributors to theanine production in Camellia sinensis. J Agric Food Chem. 2019;67:10685-93.

9. Ferreyra MLF, Rius SP, Casati P. Flavonoids: biosynthesis, biological functions, and biotechnological applications. Front Plant Sci. 2012;3:222.

10. Sarbu LG, Bahrin LG, Babii C, Stefan M, Birsa ML. Synthetic flavonoids with antimicrobial activity: a review. J Appl Microbiol. 2019;127:1282-90.

11. Daglia M, Papetti A, Grisoli P, Aceti C, Spini V, Dacarro C, et al. Isolation, identification, and quantification of roasted coffee antibacterial compounds. J Agric Food Chem. 2007;55:10208-13.

12. Islam N, Choi J, Baek K-H. Antibacterial activities of endophytic bacteria isolated from Taxus brevifolia against foodborne pathogenic bacteria. Foodborne Pathog Dis. 2018;15:269-76.

13. Schoefer L, Mohan R, Schwiertz A, Braune A, Blaut M. Anaerobic degradation of flavonoids by Clostridium orbiscindens. Appl Environ Microbiol. 2003;69:5849-54.

14. Gummadi SN, Bhavya B, Ashok N. Physiology, biochemistry and possible applications of microbial caffeine degradation. Appl Microbiol Biotechnol. 2012;93:545-54.

15. Zhang Y, Skaar I, Sulyok M, Liu X, Rao M, Taylor JW. The Microbiome and metabolites in fermented Pu-erh Tea as revealed by high-throughput sequencing and quantitative multiplex metabolite analysis. PLoS ONE 2016;11:e0157847.

16. de Santi Ferrara FI, Oliveira ZM, Gonzales HHS, Floh EIS, Barbosa HR. Endophytic and rhizospheric enterobacteria isolated from sugar cane have different potentials for producing plant growth-promoting substances. Plant Soil. 2012;353:409-17.

17. Chi F, Shen S-H, Cheng H-P, Jing Y-X, Yanni YG, Dazzo FB. Ascending migration of endophytic rhizobia, from roots to leaves, inside rice plants and assessment of benefits to rice growth physiology. Appl Environ Microbiol. 2005;71:7271-8.

18. Hirsch PR, Mauchline TH. Who's who in the plant root microbiome? Nat Biotechnol. 2012;30:961.

19. Agler MT, Ruhe J, Kroll S, Morhenn C, Kim S-T, Weigel D, et al. Microbial hub taxa link host and abiotic factors to plant microbiome variation. PLoS Biol. 2016;14:e1002352.

20. Carlström Cl, Field CM, Bortfeld-Miller M, Müller B, Sunagawa S, Vorholt JA. Synthetic microbiota reveal priority effects and keystone strains in the Arabidopsis phyllosphere. Nat Ecol Evol. 2019;3:1445-54.

21. Thiergart T, Durán P, Ellis T, Vannier N, Garrido-Oter R, Kemen E, et al. Root microbiota assembly and adaptive differentiation among European Arabidopsis populations. Nat Ecol Evol. 2020;4:122-31. 
22. Zhang J, Liu Y-X, Zhang N, Hu B, Jin T, Xu H, et al. NRT1.1B is associated with root microbiota composition and nitrogen use in field-grown rice. Nat Biotechnol. 2019;37:676-84.

23. Chen S, Waghmode TR, Sun R, Kuramae EE, Hu C, Liu B. Root-associated microbiomes of wheat under the combined effect of plant development and nitrogen fertilization. Microbiome. 2019;7:136.

24. Geddes BA, Paramasivan P, Joffrin A, Thompson AL, Christensen K, Jorrin B, et al. Engineering transkingdom signalling in plants to control gene expression in rhizosphere bacteria. Nat Commun. 2019;10:3430.

25. Walters WA, Jin Z, Youngblut N, Wallace JG, Sutter J, Zhang W, et al. Large-scale replicated field study of maize rhizosphere identifies heritable microbes. Proc Natl Acad Sci U S A. 2018;115:7368-73.

26. Kudjordjie EN, Sapkota R, Steffensen SK, Fomsgaard IS, Nicolaisen M. Maize synthesized benzoxazinoids affect the host associated microbiome. Microbiome. 2019;7:59.

27. Sharaf H, Rodrigues RR, Moon J, Zhang B, Mills K, Williams MA. Unprecedented bacterial community richness in soybean nodules vary with cultivar and water status. Microbiome. 2019;7:63.

28. Xu J, Zhang $\mathrm{Y}$, Zhang $\mathrm{P}$, Trivedi $\mathrm{P}$, Riera N, Wang $\mathrm{Y}$, et al. The structure and function of the global citrus rhizosphere microbiome. Nat Commun. Nature Publishing Group; 2018;9:1-10.

29. Horton MW, Bodenhausen N, Beilsmith K, Meng D, Muegge BD, Subramanian S, et al. Genome-wide association study of Arabidopsis thaliana leaf microbial community. Nat Commun. Nature Publishing Group; 2014;5:1-7.

30. Edgar RC. UPARSE: highly accurate OTU sequences from microbial amplicon reads. Nat Methods. 2013;10:996-998.

31. Love MI, Huber W, Anders S. Moderated estimation of fold change and dispersion for RNA-seq data with DESeq2. Genome Biol. 2014;15:550.

32. Knights D, Kuczynski J, Charlson ES, Zaneveld J, Mozer MC, Collman RG, et al. Bayesian community-wide culture-independent microbial source tracking. Nat Methods. Nature Publishing Group; 2011;8:761-3.

33. Love MI, Huber W, Anders S. Moderated estimation of fold change and dispersion for RNA-seq data with DESeq2. Genome Biol. 2014;15:550.

34. Ning D, Deng Y, Tiedje JM, Zhou J. A general framework for quantitatively assessing ecological stochasticity. Proc Natl Acad Sci U S A. 2019;116:16892-8.

35. Pollard KS, Dudoit S, Laan MJ van der. Multiple Testing Procedures: R multtest Package and Applications to Genomics, in Bioinformatics and Computational Biology Solutions Using R and Bioconductor. 2005;

36. Feizi S, Marbach D, Médard M, Kellis M. Network deconvolution as a geod to distinguish direct dependencies in networks. Nat Biotechnol. 2013;31:726-733.

37. Luo F, Zhong J, Yang Y, Scheuermann RH, Zhou J. Application of random matrix theory to biological networks. Phys Lett A. 2006;357:420-423.

38. Csardi G, Nepusz T. The igraph software package for complex network research. InterJournal. Complex Systems 2006;1695

39. R Core Team. R: A language and environment for statistical computing. R Foundation for Statistical Computing, http://wwww.R-project.org. 2019.

40. Whipps JM, Hand P, Pink D, Bending GD. Phyllosphere microbiology with special reference to diversity and plant genotype. J Appl Microbiol. 2008;105:1744-55.

41. Dini-Andreote F, Stegen JC, van Elsas JD, Salles JF. Disentangling mechanisms that mediate the balance between stochastic and deterministic processes in microbial succession. Proc Natl Acad Sci U S A 2015;112:E1326-1332.

42. Gonze D, Raes J, Faust K, Lahti L. Multi-stability and the origin of microbial community types. ISME J. 2017;11:2159.

43. Vorholt JA. Microbial life in the phyllosphere. Nat Rev Microbiol. 2012;10:828-40.

44. Chase JM, Myers JA. Disentangling the importance of ecological niches from stochastic processes across scales. Philos Trans R Soc Lond B Biol Sci. 2011;366:2351-63.

45. Vellend M. Conceptual synthesis in community ecology. Q Rev Biol. 2010;85:183-206.

46. Fierer N, Strickland MS, Liptzin D, Bradford MA, Cleveland CC. Global patterns in belowground communities. Ecol Lett. 2009;12:1238-49.

47. Griffiths RI, Thomson BC, James P, Bell T, Bailey M, Whiteley AS. The bacterial biogeography of British soils. Environ Microbiol. 2011;13:1642-1654.

48. Ma B, Lv X, Cai Y, Chang SX, Dyck MF. Liming does not counteract the influence of long-term fertilization on soil bacterial community structure and its co-occurrence pattern. Soil Biol Biochem. 2018;123:45-53.

49. Fierer N. Embracing the unknown: disentangling the complexities of the soil microbiome. Nat Rev Microbiol. 2017;15:579-90.

50. Ahkami AH, Allen White R, Handakumbura PP, Jansson C. Rhizosphere engineering: Enhancing sustainable plant ecosystem productivity. Rhizosphere. 2017;3:233-43.

51. Blossfeld S, Gansert D. A novel non-invasive optical method for quantitative visualization of pH dynamics in the rhizosphere of plants. Plant Cell Environ. 2007;30:176-86.

52. Khan AL, Ullah I, Hussain J, Kang S-M, Al-Harrasi A, Al-Rawahi A, et al. Regulations of essential amino acids and proteomics of bacterial endophytes Sphingomonas sp. Lk11 during cadmium uptake. Environ Toxicol. 2016;31:887-96.

53. Fabiano CC, Tezotto T, Favarin JL, Polacco JC, Mazzafera P. Essentiality of nickel in plants: a role in plant stresses. Front Plant Sci. 2015;6:754

54. Röttjers L, Faust K. From hairballs to hypotheses-biological insights from microbial networks. FEMS Microbiol Rev. 2018;42:761-80.

55. Faust K, Raes J. Microbial interactions: from networks to models. Nat Rev Microbiol. 2012;10:538-50.

56. Proença DN, Francisco R, Kublik S, Schöler A, Vestergaard G, Schloter M, et al. The Microbiome of Endophytic, Wood Colonizing Bacteria from Pine Trees as Affected by Pine Wilt Disease. Sci Rep. Nature Publishing Group; 2017;7:1-9.

57. Wemheuer F, Wemheuer B, Daniel R, Vidal S. Deciphering bacterial and fungal endophyte communities in 
leaves of two maple trees with green islands. Sci Rep. Nature Publishing Group; 2019;9:1-14.

58. Ding T, Melcher U. Influences of Plant Species, Season and Location on Leaf Endophytic Bacteria Communities of Non-Cultivated Plants. PLoS ONE [Internet]. 2016 [cited 2020 Mar 31];11. Available from: https://www.ncbi.nlm.nih.gov/pmc/articles/PMC4790846/

59. Pini F, Galardini M, Bazzicalupo M, Mengoni A. Plant-Bacteria Association and Symbiosis: Are There Common Genomic Traits in Alphaproteobacteria? Genes. 2011;2:1017-32.

Figures

Figure 1 Compositions and core microbiota of microbiomes in different $\mathbf{C}$. sinensis compartments. (a) Location of sampling sites. (b) $\alpha$-diversity (Shannon-Wiener index) of microbiomes in each compartment $(n=45)$. (c) $\beta$-diversity (weighted UniFrac distance) of microbiomes in each compartment $(n=45)$. (d) Relative abundance of microbial taxa found in each compartment. (e) Unique and shared core microbiota.

Figure 2 The association of microbiomes in C. sinensis compartments. (a) Source proportions for sequential compartments; arrow direction indicates potential source of microbiomes and arrow weight varies with proportion. (b) Enrichment (positive) and depletion (negative) of OTUs between ascending compartments; red points indicate significant enriched or depleted OTUs (Wald test, $\mathrm{Pi}_{\mathrm{i}} \mathrm{0.05}$ ). (c) Genera of enriched and depleted OTUs between ascending compartments. Colors of points indicate phylum classification.

Figure 3 The contribution of stochasticity, dispersal limitation and environmental selection to microbiome community assembly processes in C. sinensis compartments. (a) Normalized stochastically ratio (NST). (b) Nestedness (NODF) at five taxon ranks. (c) Nestedness at phylum level. (d) The relationship between community similarity and geographic distance. Mantel's $r$ and $p$ values indicate the results of partial Mantel tests while controlling with the environmental matrix. (e) Mantel correlograms. Red points indicate significant Mantel's $r\left(\mathrm{Pi}_{\mathrm{i}} 0.05\right)$. (f) Constrained corresponding analysis (CCA) of compartment microbiomes; text labels indicate significant environmental drivers (permutation test for CCA, $\mathrm{Pi}_{\mathrm{i}} 0.05$ ).

Figure 4 Links and taxon assortativity in co-occurrence network of microbiomes of different C. sinensis compartments.

Figure 5 The trade-off between stochastic and deterministic processes in C. sinensis compartment microbiomes. The volume of cylinder represents the contribution of various assembly processes in corresponding microbiomes of tea plant compartments. 
Additional Files

Suplementary Materials

Table S1. The background information of sampling locations.

512 Table S2. The topological characteristics of microbial co-occurrence network for microbiomes in various

513 compartments.

514 Data files

515 Data file S1. The physicochemical properties of bulk soils.

516 Data file S2. The physicochemical properties of rhizosphere soils.

517 Data file S3. The physicochemical properties of roots.

518 Data file S4. The physicochemical properties of old leaves.

519 Data file S5. The physicochemical properties of young leaves. 

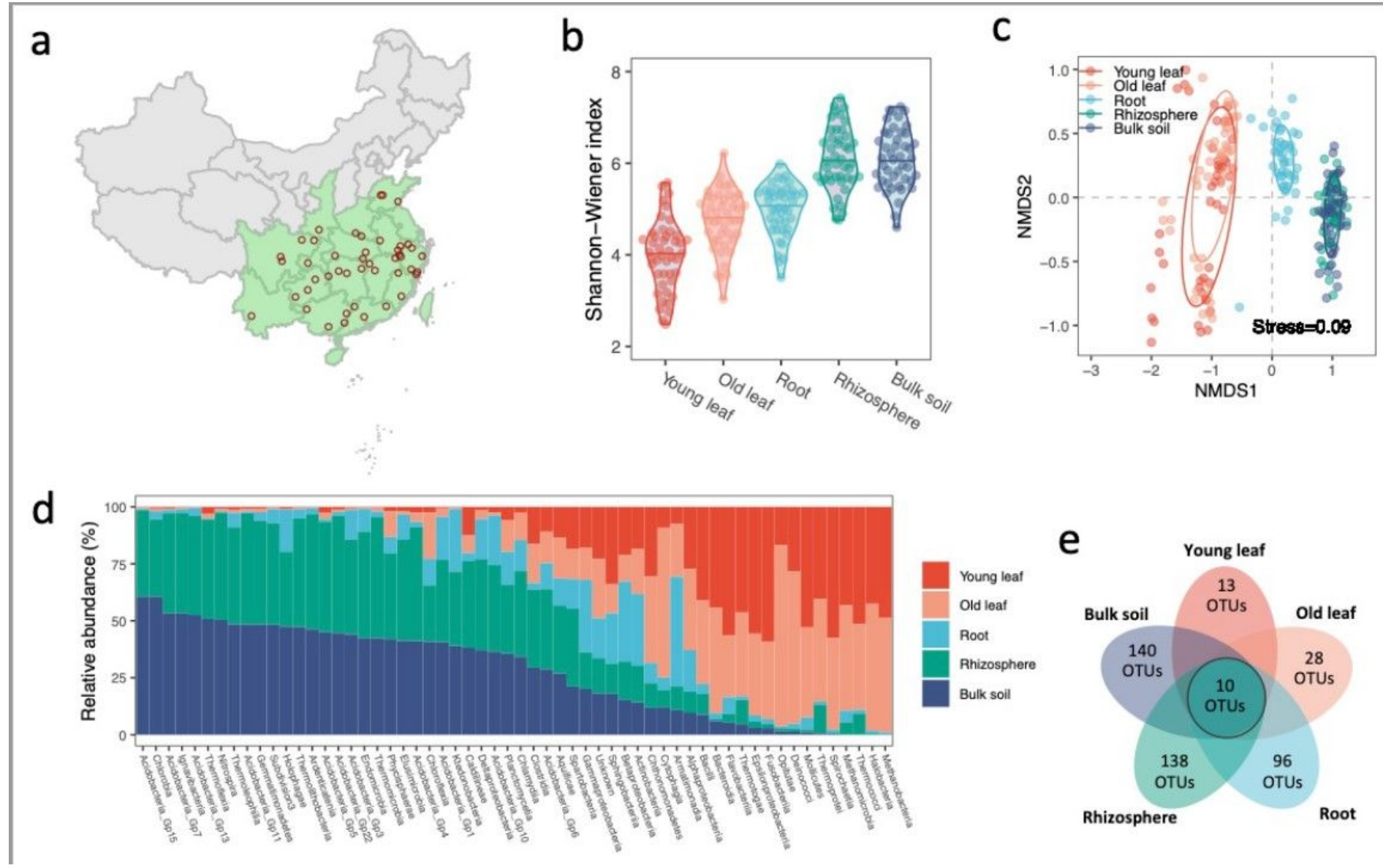

\section{Figure 1}

Compositions and core microbiota of microbiomes in di囚erent C. sinensis compartments. (a) Location of sampling sites. (b) \-diversity (Shannon-Wiener index) of microbiomes in each compartment ( $n=45)$. (c) diversity (weighted UniFrac distance) of microbiomes in each compartment $(n=45)$. (d) Relative abundance of microbial taxa found in each compartment. (e) Unique and shared core microbiota. 
a

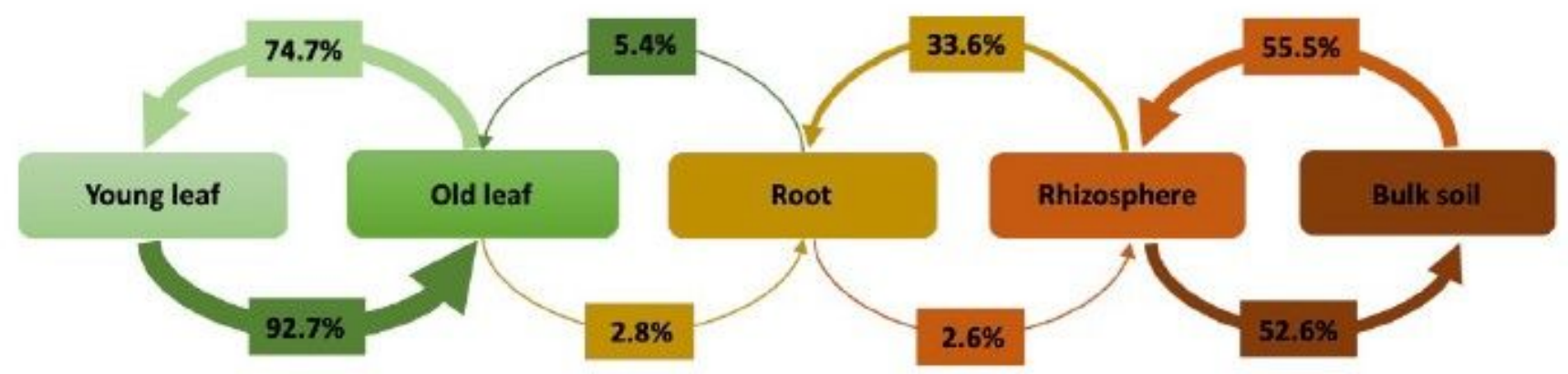

b Young leaf/Old leaf
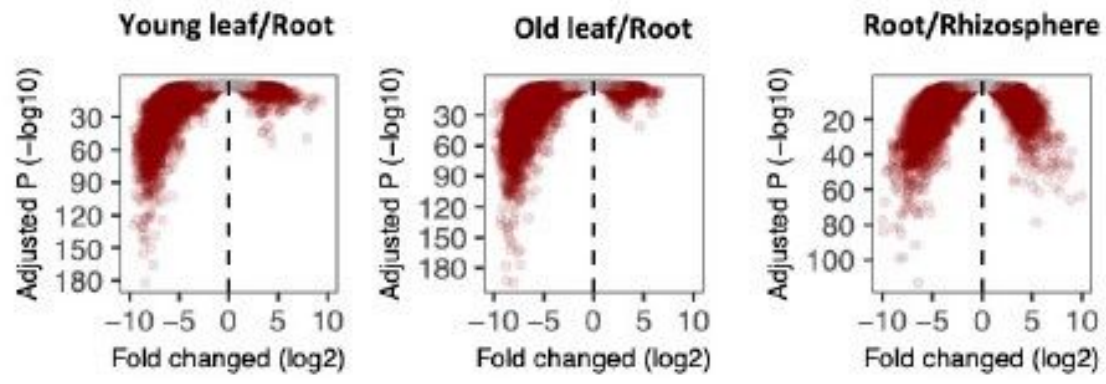

Rhizosphere/Bulk soil

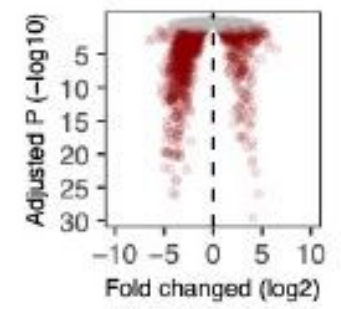

C Young leaf/Old leaf

Young leaf/Root
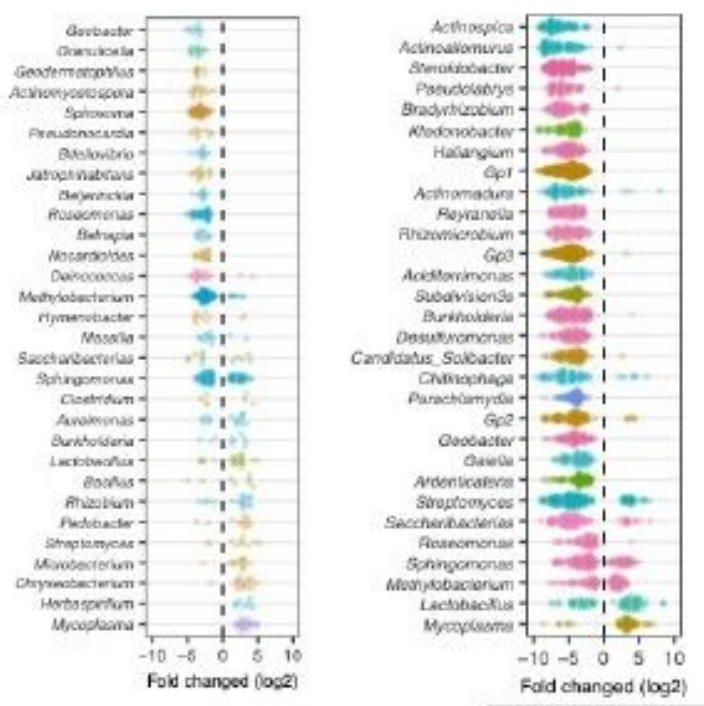

Old leaf/Root
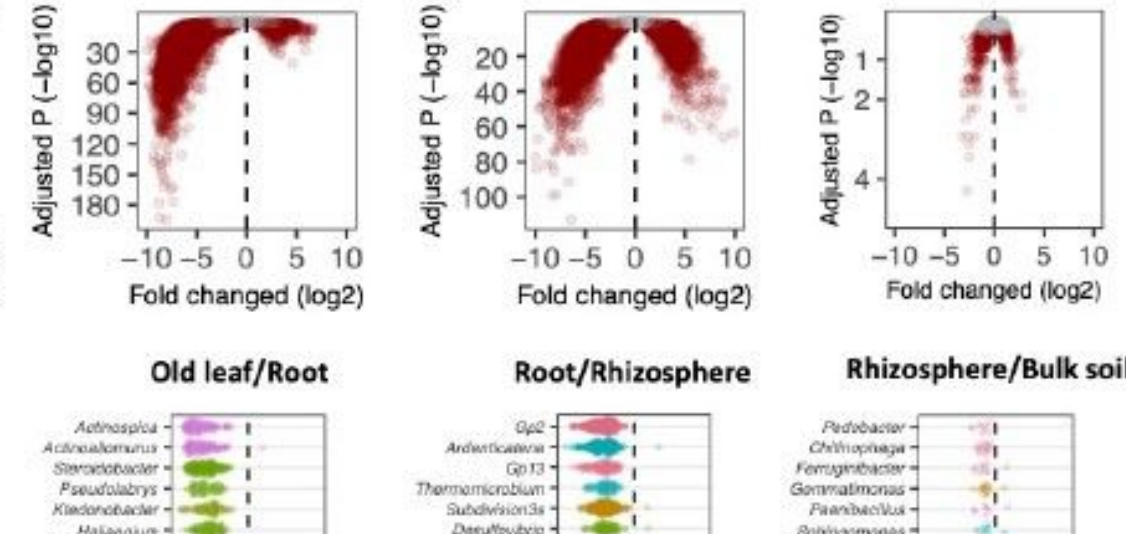

Kiasomotencier -

Macongur $=2$ !

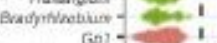

miecrvcrobur - 0 ;

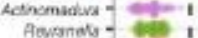

Aevarinats -

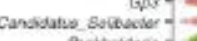

Berkboblers -

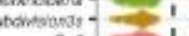

$\mathrm{Gp} 2=$

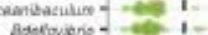

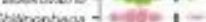

pencomenacos of

Parastharyota -

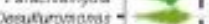

succharbuctowlas -

Anderncatars "]

Simpolaryoses -

Goccucier - 20:

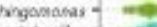

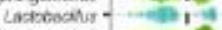

Mothwetactorium

Fold changed $\langle\log 2\}$

Fold chenged $(\log 2)$

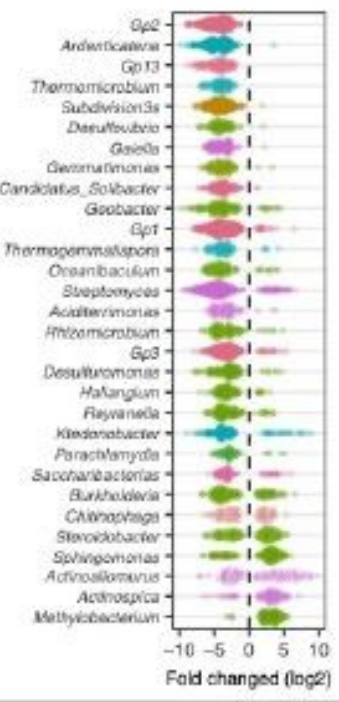

Rhizosphere/Bulk soil

- Acidobacteria

- Actinobacteria

- Chlamydiae

- Chloroflexi

- Deinococcus-Thermus

- Candidatus_Sacchanbacteria. Firmicutes

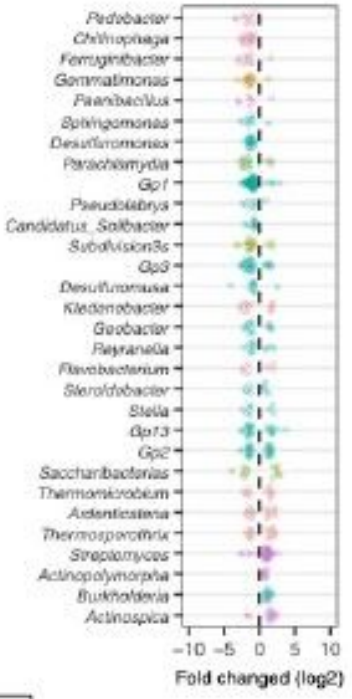

\section{Figure 2}

The association of microbiomes in C. sinensis compartments. (a) Source proportions for sequential compartments; arrow direction indicates potential source of microbiomes and arrow weight varies with proportion. (b) Enrichment (positive) and depletion (negative) of OTUs between ascending compartments; red points indicate signicant enriched or depleted OTUs (Wald test, $\mathrm{P}<0.05$ ). (c) Genera of enriched and depleted OTUs between ascending compartments. Colors of points indicate phylum classication. 
a

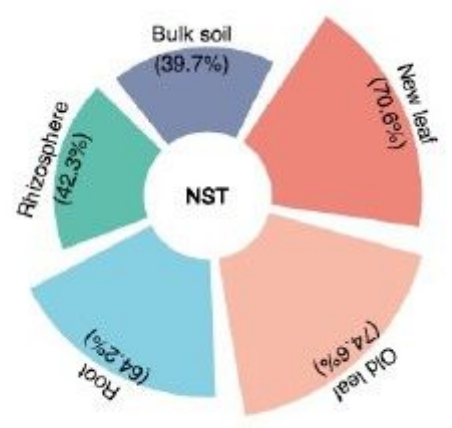

C

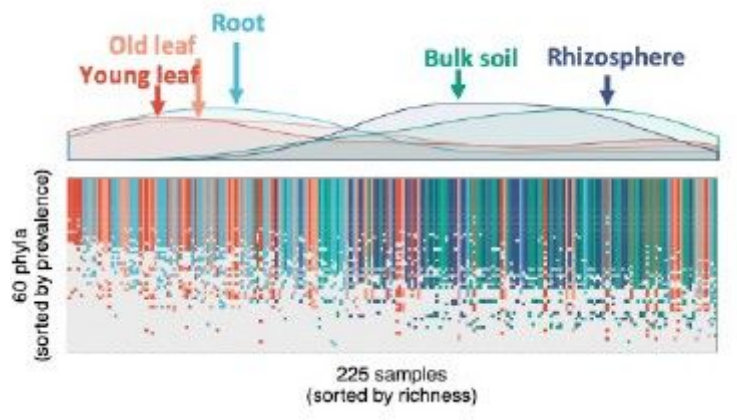

f

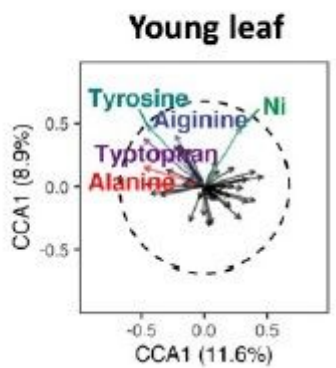

Old leaf

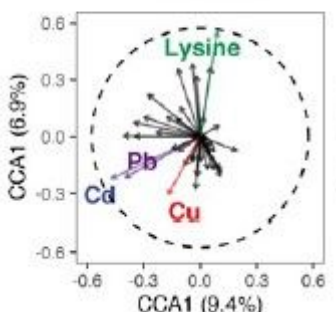

b

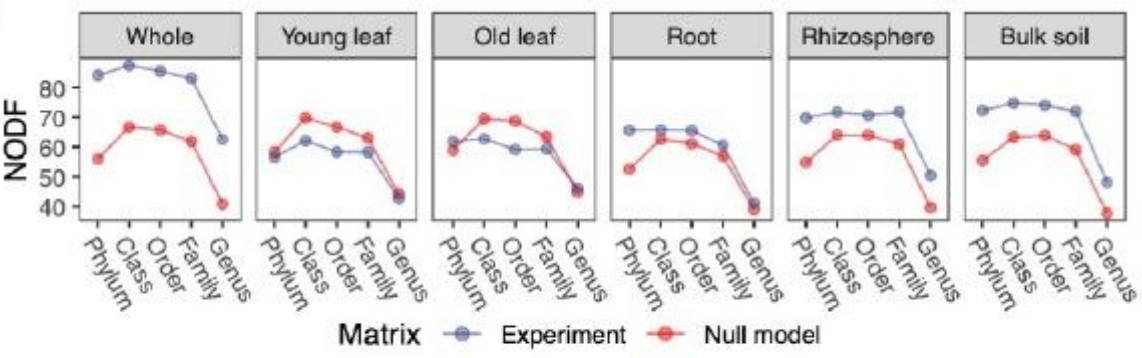

d

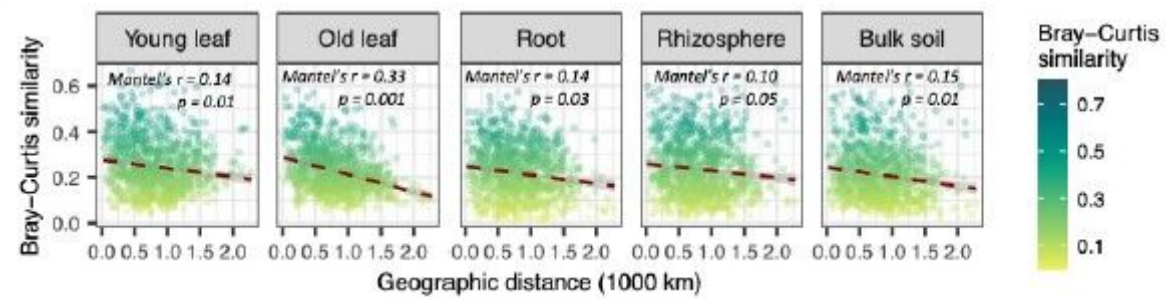

e

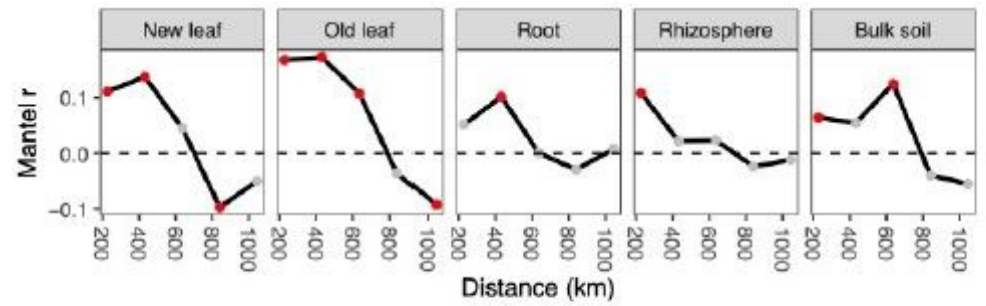

Root

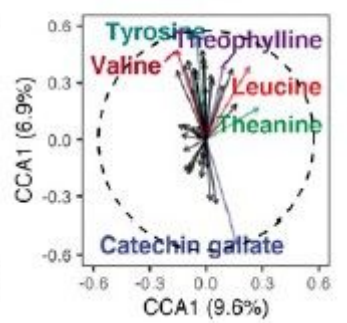

Bulk soil

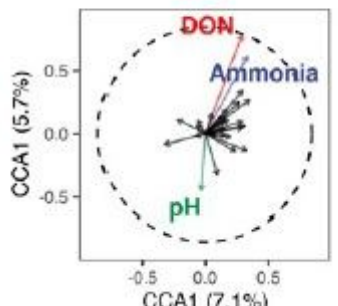

\section{Figure 3}

The contribution of stochasticity, dispersal limitation and environmental selection to microbiome community assembly processes in C. sinensis compartments. (a) Normalized stochastically ratio (NST). (b) Nestedness (NODF) at ve taxon ranks. (c) Nestedness at phylum level. (d) The relationship between community similarity and geographic distance. Mantel's $r$ and $p$ values indicate the results of partial Mantel tests while controlling with the environmental matrix. (e) Mantel correlograms. Red points indicate signicant Mantel's $r(P<0.05)$. (f) Constrained corresponding analysis (CCA) of compartment microbiomes; text labels indicate signicant environmental drivers (permutation test for CCA, $\mathrm{P}<0.05$ ). 


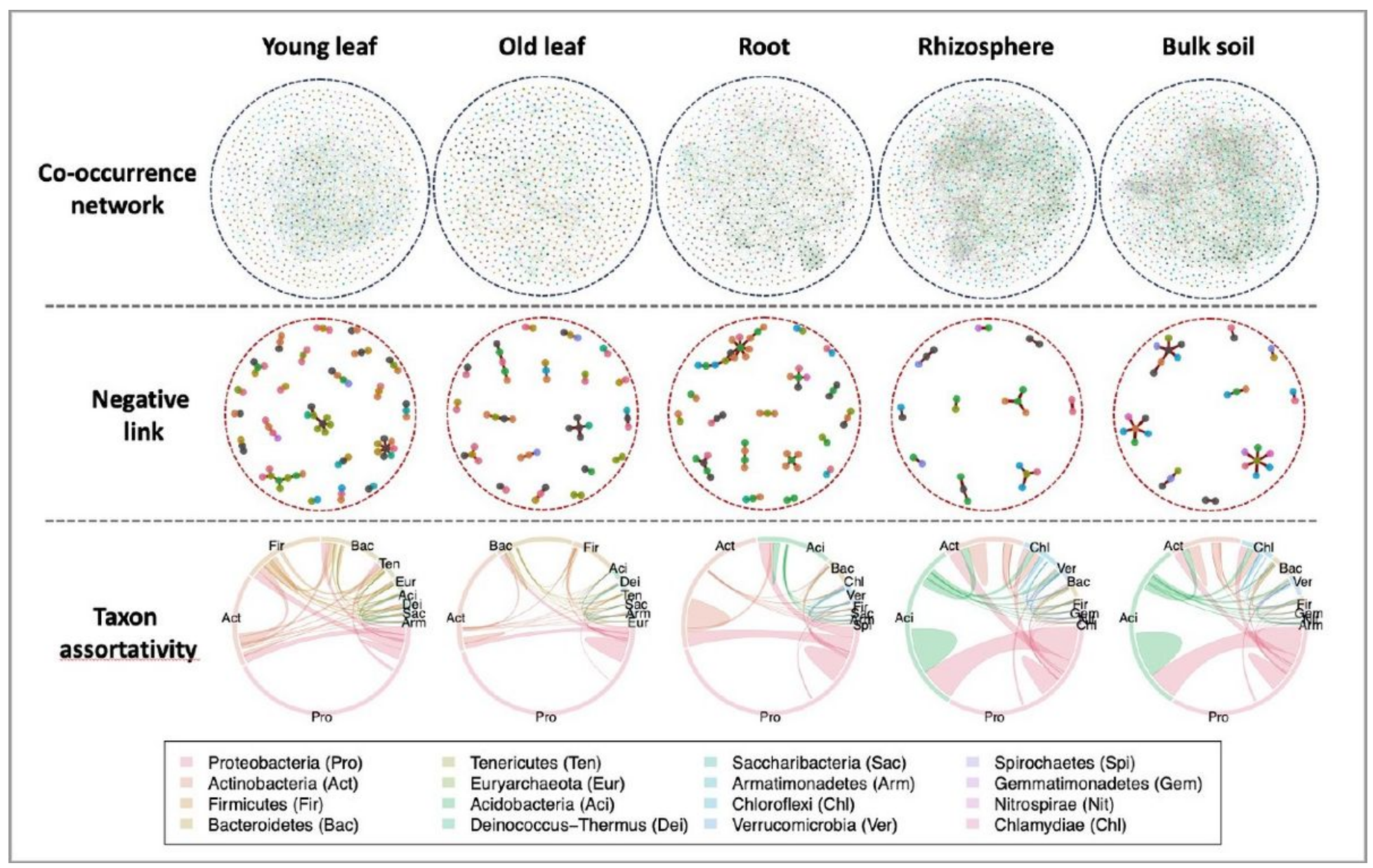

\section{Figure 4}

Links and taxon assortativity in co-occurrence network of microbiomes of different $\mathrm{C}$. sinensis compartments. 


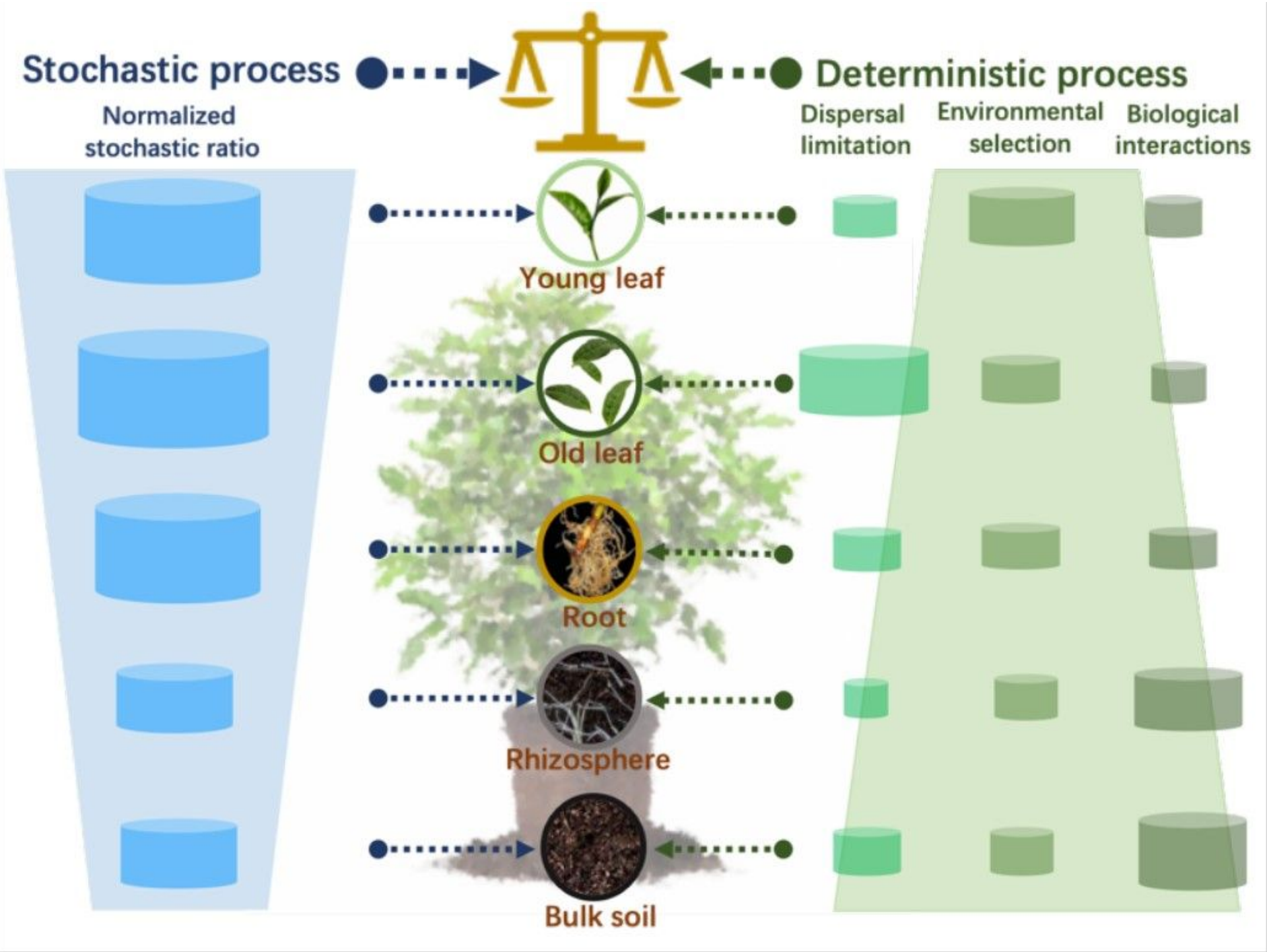

\section{Figure 5}

The trade-off between stochastic and deterministic processes in C. sinensis compartment microbiomes. The volume of cylinder represents the contribution of various assembly processes in corresponding microbiomes of tea plant compartments.

\section{Supplementary Files}

This is a list of supplementary files associated with this preprint. Click to download.

- TableS2.xlsx

- TableS6.xlsx

- TableS3.xlsx

- TableS4.xIsx

- TableS.docx 
- Tables5.xIsx 